1 Evaluation of small non-coding RNAs as a possible epigenetic mechanism mediating the transition from

2 biotrophy to necrotrophy in the life cycle of Phytophthora infestans

4 Juliana González-Tobón ${ }^{*}, 1,2$, Alejandra Rodríguez-Jaramillo ${ }^{1}$, Laura Milena Forero ${ }^{1}$, Laura Natalia González ${ }^{1}$,

5 Giovanna Danies ${ }^{3}$, and Silvia Restrepo ${ }^{4}$

7 Address: ${ }^{1}$ Department of Biological Sciences, Universidad de los Andes, Kr. 1 \# 18A 12, Bogotá, Colombia.

$8{ }^{2}$ School of Integrative Plant Science, Plant Pathology and Plant-Microbe Biology Section, Cornell University,

9 Ithaca, NY, U.S.A. ${ }^{3}$ Department of Design, Universidad de los Andes, Kr. 1 \# 18A 12, Bogotá, Colombia.

$10{ }^{4}$ Department of Food and Chemical Engineering, Universidad de los Andes, Kr. 1 \# 18A 12, Bogotá, Colombia.

11

12 Email: J González-Tobón* - j.gonzalez10@uniandes.edu.co; A Rodríguez-Jaramillo -

13 a.rodriguez16@uniandes.edu.co; LM Forero - $\underline{\operatorname{lm} . f o r e r o 10 @ u n i a n d e s . e d u . c o ; ~ L N ~ G o n z a ́ l e z ~-~}$

14 In.gonzalez138@uniandes.edu.co; G Danies - g-danies@uniandes.edu.co; S Restrepo - $\underline{\text { srestrep@ uniandes.edu.co }}$

$15 *$ Corresponding author.

16

17 ORCID ID: 0000-0002-5684-4371 (J González-Tobón); ORCID ID: 0000-0002-4079-8956 (L N González);

18 ORCID ID: 0000-0001-9689-1140 (G Danies); ORCID ID: 0000-0001-9016-1040 (S Restrepo)

\title{
22 Acknowledgments
}

23 We thank Jose A. Vargas for his valuable advice during the development of this work. 


\section{Summary}

26 Phytophthora infestans, causal agent of late blight disease of potatoes, causes billion-dollar losses worldwide each

27 year. This plant pathogen is a hemibiotroph, first feeding on the host and later killing it. Even though the transcription

28 dynamics of this transition are characterized, the role that small non-coding RNAs (sRNAs) might have is still

29 unknown. Furthermore, a bioinformatic pipeline to search and analyze sRNAs in P. infestans, is needed. Using our

30 proposed pipeline, 146 sRNAs were found to be significantly differentially expressed between the evaluated stages of

31 the pathogen's life cycle. One hundred of these sRNAs were successfully annotated and classified into nine functional

32 categories. The expression of the genes associated to ten of these sRNAs was validated via qRT-PCR. Among these,

33 the expression levels of genes encoding for effectors were inversely correlated to that of the sRNAs aligning to them,

34 which is expected if sRNAs are indeed regulating their expression. This correlation was not clear for sRNAs in other

35 functional categories and should not be confused with strict causality. This study works as a starting point for

36 considering sRNAs as role players in the transition from biotrophy to necrotrophy in P. infestans when infecting

\section{Solanum tuberosum.}

\section{Keywords:}

40 Epigenetics, small non-coding RNAs, microbiology, computational biology, Phytophthora infestans 
Phytophthora infestans is a devastating plant pathogenic oomycete that causes late blight disease of tomatoes and potatoes (Schoina \& Govers 2015). This pathogen is a hemibiotroph, first feeding on living host tissue (biotrophy)

44 and then continuing to live in dead tissue (necrotrophy) (Fry 2008). It is unclear how and why this transition occurs,

45 but it seems to be related with the secretion of effector proteins by the pathogen to the host, which in turn responds using resistance genes (R genes). Some effectors are expressed early in the infection cycle and others later, obeying

47 the "accelerator and brake" model, which states that they can either slow down (brake) or accelerate necrosis

48 (Zuluaga et al. 2016; Lee \& Rose 2010). This creates a coevolutionary arms race between the pathogen and the host.

Gaining or losing an effector can happen in a stable way (mutations) or through flexible mechanisms

50 (epigenetic regulation) (Gijzen et al. 2014; Kasuga \& Gijzen 2013). This latter option regulates the expression of a

51 transcript without altering the gene's sequence, enabling the gene to function in the future. Moreover, this is a fast

52 response that gives the pathogen a plethora of opportunities to respond to its environment. The most studied

53 epigenetic mechanism of $P$. infestans are small non-coding RNAs (sRNAs) (Kasuga \& Gijzen 2013; Moazed 2009).

54 These are taught to be involved in transposon control and their length usually ranges between 19-40 bp (varying

55 among species) (Jia et al. 2017; Vetukuri et al. 2012; Fahlgren et al. 2013). Phytophthora infestans' genome is

56 repetitive in $75 \%$ of its length and sRNAs have been found immersed in these regions. Most interestingly, they

57 usually overlap effector genes (Jia et al. 2017; Vetukuri et al. 2012; Haas et al. 2009). Therefore, it has been

58 hypothesized that they could silence/activate the effectors' expression by altering how heterochromatized

59 (condensed) these regions are (Gijzen et al. 2014; Moazed 2009; Vetukuri et al. 2012).

60 The transition from biotrophy to necrotrophy has not been studied much under the light of epigenetics.

61 Available literature on sRNAs focus on their specific types and evolutionary traces in the genome (Bollmann et al.

62 2016). This may be due to the lack of clear bioinformatic pipelines to analyze this type of data in P. infestans.

63 Available pipelines are usually designed for animal or plant data (Rueda et al. 2015; Giurato et al. 2013; Gupta et al.

64 2012). The aim of this study was to evaluate whether sRNAs can constitute an epigenetic regulatory mechanism for

65 the transition from biotrophy to necrotrophy in P. infestans. Additionally, this phenomenon was used as a model to

66 design a bioinformatic pipeline that can be used for the analysis of sRNA data sets in this plant pathogen.

67 The pipeline used is depicted in Figure 1. Our first step was to acquire data (grey bar, Fig. 1). For this, we searched extensively for small RNA-Seq data exclusively from $P$. infestans, whose sequencing libraries did not 69 remove sRNAs, and that included samples of different stages in the pathogen's life cycle. Only one data set fulfilled 
these requirements: Asman and collaborators (2014) (Åsman et al. 2014) (GEO accession: GSE63292). The authors

71 briefly inoculated leaves of Solanum tuberosum with P. infestans and extracted sRNAs at 24, 48, and 72 hours post

72 inoculation (hpi), as well as from mycelia growing on culture media. For their isolate (88069), 24 hpi represented the

73 biotrophic stage, 48 hpi the transition, and 72 hpi the necrotrophic stage ( $̊$ sman et al. 2014).

To process this raw data, we performed two big steps: trimming and selection (represented by orange and

75 blue bars in Fig. 1, respectively). We first used Fastq-dump and FastQC to download the data from NCBI and

76 convert it from SRA files to FASTQ format. The reads exhibited very good quality scores (> 40 Phred score) in all

77 base pairs except for the last one, which was removed using cutadapt (Martin 2011). This process was performed

78 only on reads that contained adaptor sequences, which were also removed. Then, only those reads between 18-30 bp

79 were selected using cutadapt to eliminate sequences that were not adequately processed (Vetukuri et al. 2012;

80 Fahlgren et al. 2013; Åsman et al. 2014). All rRNAs (ribosomal RNAs) and tRNAs (transfer RNAs) (Vetukuri et al.

81 2012) were removed to limit the analyses only to regulatory RNA species. Known rRNA and tRNA sequences of $P$.

82 infestans were downloaded from Ensembl Protists and reads were aligned using Bowtie1 (Langmead et al. 2009).

83 The parameters for these alignments were: only report the first valid alignment (-k 1) and allow 0 mismatches. The

84 resulting reads were aligned to the S. tuberosum genome in the same way.

85 Finally, the reads were ready to be analyzed (represented by the purple bar in Fig. 1). They were aligned to

86 the T30-4 P. infestans genome from the Broad Institute using Bowtie 1 with the same parameters mentioned above

87 (Vetukuri et al. 2012; Fahlgren et al. 2013; Åsman et al. 2014; Åsman et al. 2016). A GTF file with the annotation of

88 P. infestans genes was obtained from Ensembl Protists. This file, as well as the alignment files, were used as input

89 for HTSeq (Anders et al. 2015) to generate count tables that indicated how many times each sRNA was aligned to

90 the genome. These tables were the input for DESeq2 (Love et al. 2014) to determine differentially expressed sRNAs

91 between samples. DESeq2 normalized these counts via the Relative Log Expression (RLE) method (Reddy 2015).

92 This analysis was performed in Galaxy online (Afgan et al. 2016), where the factor specified was "hpi" with four

93 levels: $24,48,72$, and mycelia in culture. The threshold for p-values associated with the $\log _{2}$ fold change (FC) of

94 each sRNA was fixed at 0.05 and each significantly differentially expressed sRNA was annotated using Blast2GO

95 and classified into a functional category. 
A set of 146 sRNAs was found to be significantly differentially expressed among the four samples. $\log _{2}$ fold changes for upregulated sRNAs ranged between $2.8-4.4$, and for downregulated sRNAs between -2 and -4.25 . Out

99 of these, six were identified as tRNAs and pseudo tRNAs, and one as a 28S rRNA. Additionally, thirty-nine ncRNAs were associated with putative uncharacterized proteins. The remaining 100 sRNAs were successfully annotated to specific genes and then classified into nine functional categories that are summarized in Figure 2. The first three categories include sRNAs associated with non-protein-coding genes and the remaining ones contain sRNAs mapping to protein-coding genes. Three of the latter included effectors, which are known to be directly associated with the transition from biotrophy to necrotrophy. Therefore, we mostly focused on these three categories for further analyses. A principal component analysis (PCA) among the four individual data sets is shown in Figure 3. As expected, mycelia grown in media differed considerably from any of the other data sets, more than did any of the other data sets between each other. Interestingly, data from $48 \mathrm{hpi}$ and $72 \mathrm{hpi}$ were much more similar between them than when compared to the 24 hpi data set.

To gather further experimental evidence on our predicted sRNAs actually regulating the expression of their corresponding genes during the transition from biotrophy to necrotrophy, the expression of ten of these genes (Table

111 1) was tested via a quantitative real-time reverse transcription PCR (qRT-PCR). Solanum tuberosum plants of the

112 Diacol Capiro variety were grown for one month and a half under greenhouse conditions. Then, they were inoculated 113 with $P$. infestans isolate RC1\#10 (EC-1 clonal lineage). The isolate was grown in pea agar for seven days at $\pm 20^{\circ} \mathrm{C}$.

114 Mycelia was scraped to obtain $1 \mathrm{~mL}$ of sporangial suspension and diluted to $1 \times 10^{3}$ sporangia $\mathrm{mL}^{-1}$. Two $10-\mu \mathrm{L}$ drops 115 of the sporangial suspension were placed on each side of the main vein of the leaf, abaxial side up. Three leaflets per 116 petri dish were maintained in moist chambers until symptoms developed. Subsequently, two transfers to healthy 117 leaflets were performed by immersing the diseased leaves into distilled water and adjusting the solution to $1 \times 10^{3}$ 118 sporangia $\mathrm{mL}^{-1}$ and repeating the inoculation process. This was done to obtain sporangia that came directly from the 119 leaf and not from medium Plant tissue was sampled at $12 \mathrm{hpi}$ (biotrophic stage), $48 \mathrm{hpi}$ (transition from biotrophy to necrotrophy), and

$12196 \mathrm{hpi}$ (necrotrophic stage). These time points were determined specifically for this isolate and potato variety, under 122 the assessed conditions. The area where each droplet was placed was cut out and frozen at $-80{ }^{\circ} \mathrm{C}$. Sporangia from 123 mycelia growing in pea agar were collected as a control and stored at $-80^{\circ} \mathrm{C}$. Total RNA was extracted from these 124 using the RNeasy Plant Mini Kit (QIAGEN, Hilden, Germany) following the manufacturer's instructions. RNA 
125 quality was assessed in an agarose gel and quantity was determined using a NanoDrop 1000 Spectrophotometer 126 (Thermo Fisher Scientific, Waltham, MA, USA). Subsequently, extracted RNA was converted to cDNA using 127 RevertAid H Minus First Strand cDNA Synthesis Kit (ThermoFisher), following the manufacturer's instructions.

128 Total transcript levels were determined using the Luna Universal qPCR Master Mix (2X) (New England 129 BioLabs, Ipswich, MA, USA), following the manufacturers' protocol. Gene primers were designed exon to exon using 130 Primer-BLAST software (Ye et al. 2012) (Table 1). The P. infestans 40S ribosomal protein S3A (PITG_04425) was 131 used as the constitutively expressed endogenous control as suggested by Yan \& Liou (2006). All genes were assayed 132 in triplicate in MicroAmp ${ }^{\mathrm{TM}}$ Fast Optical 96-Well Reaction Plates (Applied Biosystems, Foster City, CA, USA), and 133 three biological replicates of each treatment were performed. A control lacking template and a control lacking reverse 134 transcriptase were included. RNA from P. infestans grown in culture was used as the calibrator. The 7500 Fast Real135 Time PCR System (Applied Biosystems, Foster City, CA, USA) was used and results were analyzed using the REST 136 software (Pfaffl et al. 2002). Results of these qRT-PCR profiles are summarized in Figure 4.

137 Three main functional groups were studied in detail: effector-related genes, enzymatic processes and 138 transporter-like proteins. Among the effector genes, one RxLR effector and two elicitins (INF1 \& INF6) were tested. 139 The Avr2 family secreted RxLR effector was upregulated during biotrophy and downregulated later in the cycle. 140 Inversely, its sRNA was significantly upregulated at the necrotrophic stage. No other significant differences in its 141 expression were detected during the rest of the cycle (see Figure 5). Since, RxLR effectors are almost always related 142 to the biotrophic stage of the infection this finding was expected (Zuluaga et al. 2016). Furthermore, these results 143 support the proposition that the RxLR sRNA is modulating the gene's expression by the end of the infection cycle. 144 Similarly, sRNAs associated to crinkler and crinkler-like proteins, usually related to necrotrophy in $P$. infestans, were 145 downregulated at the later stages of infection. This supports the idea that the corresponding genes are generally 146 expressed during these later stages (Zuluaga et al. 2016, Stam et al. 2013). However, the expression of the crinkler 147 genes we used in this study, was not validated using qRT-PCR. Finally, elicitins and elicitin-like proteins are relevant 148 effectors expressed at different time points of the infection. Some are known to be highly expressed during biotrophy 149 while others are highly expressed during necrotrophy (Zuluaga et al. 2016). INF-1, INF-6 and INF-2A are defined as 150 important necrotrophic elicitors (Zuluaga et al. 2016; Kanneganti et al. 2006) and as inducers of various degrees of 151 the hypersensitive response or programmed cell death in plants (Kamoun 2001). However, INF-1 and INF-6 genes 
152 were downregulated at all stages of the cycle. The sRNAs associated to them, and to INF-2A, showed the same pattern, 153 thus not supporting the expected inverse correlation (see Figure 5).

154 Expression patterns of sRNAs and genes related to enzymatic processes support the regulatory role of sRNAs 155 in most cases (see Figure 5). Two enzymes associated with sporulation and thus expected to express by the end of the 156 infection cycle were tested: a sporangia-induced phosphatidyl inositol kinase and a nuclear LIM spore-specific factor

157 (Tani \& Judelson 2006, Judelson \& Tani 2007). Only for the kinase gene an expected inverse pattern of expression 158 was detected when compared to its corresponding sRNA during biotrophy (see Figure 5). There was no significant differential expression of this sRNA at other points of the cycle. This could indicate that the sRNA performs a

160 suppressing role at the beginning of the cycle when sporulation has not yet occurred. An inverse pattern was not 161 evident for the LIM spore-specific factor. Additionally, a gene encoding a peptidyl-prolyl cis-trans isomerase also 162 presented an inverse profile when compared with its sRNA during biotrophy (see Figure 5). This highlights the 163 complexity of the disease progression and the regulation of genes involved in it, a process where sRNAs seem to be 164 involved only partly on it.

As for transporters across the cell membrane, we also identified an inverse relation between sRNAs' and genes' expression when evaluating a berberine-like protein and an ATP-binding cassette (ABC) transporter.

167 Berberine-like proteins are known to be involved in alkaloid biosynthesis and hydrogen peroxide production. Hence, 168 they are important virulence factors expected to help in disease progression (Meijer et al. 2014). In the same sense, 169 ATP-binding cassette (ABC) transporters are involved in detoxification which allow the pathogen to manage host's 170 defenses mostly in the form of reactive oxygen species (ROS) (Zuluaga et al. 2016). We did observe an inverse pattern 171 of expression between these genes and their corresponding sRNAs (see Figure 5). For the Berberine-like protein this 172 was observed late in the infection cycle (necrotrophy) and for the ABC transporter earlier in the infection cycle 173 (biotrophy and hemibiotrophy). Finally, the genes for a haustorium-specific membrane protein and a second ABC 174 transporter did not present differential expression in the qRT-PCR assays performed. Testing a larger number of ABC 175 transporters as well as of other transporters and enzymes, might be necessary to understand their expression profiles 176 with more precision.

177 The expression of sRNAs corresponding to effector genes in $P$. infestans seems to be inversely correlated 178 with these genes' transcription. This would support the hypothesis of regulatory sequences such as sRNAs performing 179 a role during the pathogen's transition from biotrophy to necrotrophy. This correlation should not be understood as 
180 strict causality since the differential expression observed for these sRNAs could be either a cause or a consequence,

181 which must be further investigated. Moreover, it is important to note that the role of these sRNAs as modulators of

182 specific genes related to infection does not seem to be constant across all the infection cycle but rather localized to

183 certain points within it. Furthermore, sRNAs seem to be involved only partly in regulating the expression of genes

184 related to enzymatic and transportation processes during the disease cycle. However, this study works as a starting

185 point for considering sRNAs as role players in the transition from biotrophy to necrotrophy in $P$. infestans when

186 affecting S. tuberosum.

187

\section{Declarations}

189 Funding

190 No funding was received for this study.

191

\section{Competing interests}

193 The authors declare that they have no competing interests.

\section{Ethics approval}

196 Not applicable.

\section{Consent to participate}

199 Not applicable.

\section{Consent for publication}

202 Not applicable.

\section{Availability of data and materials}

205 The data sets generated and/or analyzed in the current study are available in the GitHub repository 206 https://github.com/jgonzalez10/Small-ncRNAs-pipeline 


\section{Code availability}

Code is available in the GitHub repository https://github.com/jgonzalez10/Small-ncRNAs-pipeline

\section{Author contributions}

212 JG-T developed and implemented the proposed pipeline, analyzed the results, performed all the inoculation steps, the

213 qRT-PCR experiments, and wrote the manuscript. AR-J helped perform the qRT-PCR experiments and the inoculation

214 steps. LMF implemented the pipeline and contributed significantly to the bioinformatic analyses. LNG provided

215 general guidance for the development of the study, specifically for the bioinformatic pipeline implementation. GD

216 provided general guidance for the development of the study and made substantial contributions to writing and editing

217 of the manuscript. SR provided continuous and profound guidance on the development of the manuscript, analyzed

218 the results and was a major contributor in writing the manuscript. All authors read and approved the final manuscript.

\section{References}

221 1. Afgan E, Baker D, van den Beek M, Blankenberg D, Bouvier D, Čech M et al (2016) The Galaxy platform for accessible, reproducible and collaborative biomedical analyses. Nucleic Acids Res 44:W3-10.

2. Anders S, Pyl PT, Huber W (2015) HTSeq — a Python framework to work with high-throughput sequencing data. Bioinformatics 31:166-9.

3. Åsman AKM, Vetukuri R, Jahan SN, Fogelqvist J, Corcoran P, Avrova AO et al (2014) Fragmentation of tRNA in Phytophthora infestans asexual life cycle stages and during host plant infection. BMC Microbiology $14: 1-12$.

4. Åsman AKM, Fogelqvist J, Vetukuri R, Dixelius C (2016) Phytophthora infestans Argonaute 1 binds microRNA and small RNAs from effector genes and transposable elements. New Phytologist 211:993-1007. genus Phytophthora. Frontiers in plant science 7:284. 
7. Fry W (2008) Phytophthora infestans: The plant (and R gene) destroyer. Molecular Plant Pathology 9:385402.

8. Gijzen M, Ishmael C, Shrestha, SD (2014) Epigenetic control of effectors in plant pathogens. Frontiers in Plant Science 5:1-5.

9. Giurato G, De Filippo MR, Rinaldi A, Hashim A, Nassa G, Ravo M et al (2013) iMir: An integrated pipeline for high-throughput analysis of small non-coding RNA data obtained by small RNA-Seq. BMC Bioinformatics 14:1-9.

10. Gupta V, Markmann K, Pedersen CN, Stougaard J, Andersen SU (2012) shortran: a pipeline for small RNAseq data analysis. Bioinformatics 28:2698-700.

11. Haas BJ, Kamoun S, Zody MC, Jiang RHY, Handsaker RE, Cano LM et al (2009) Genome sequence and analysis of the Irish potato famine pathogen Phytophthora infestans. Nature 461:393-8.

12. Jia J, Lu W, Zhong C, Zhou R, Xu J, Liu W, ... Shan, W (2017) The 25-26 nt small RNAs in Phytophthora parasitica are associated with efficient silencing of homologous endogenous genes. Frontiers in microbiology 8:773.

13. Judelson H, Tani S (2007) Transgene-induced silencing of the zoosporogenesis-specific NIFC gene cluster of Phytophthora infestans involves chromatin alterations. Eukaryotic cell 6(7):1200-1209.

14. Kamoun S (2001) Nonhost resistance to Phytophthora: novel prospects for a classical problem. Current opinion in plant biology 4:295-300.

15. Kanneganti TD, Huitema E, Cakir C, Kamoun S (2006) Synergistic Interactions of the Plant Cell Death Pathways Induced by Phytophthora infestans Nep1-Like Protein PiNPP1.1 and INF1 Elicitin. MPMI 19:85463.

16. Kasuga T, Gijzen M (2013) Epigenetics and the evolution of virulence. Trends Microbiol 21:575-82. DNA sequences to the human genome. Genome Biology 10:R25. pathogens by secreted effector proteins. Plant Signaling \& Behavior 5:769-72. with DESeq2. Genome Biology 15:1-21. 

reads. EMBnet.journal 17:10-2.

21. Maze I, Shen L, Zhang B, Garcia BA, Shao N, Mitchell A et al (2014) Analytical tools and current challenges in the modern era of neuroepigenomics. Nature Neuroscience 17:1476-90. and extracellular proteome of the potato late blight pathogen Phytophthora infestans. Molecular \& Cellular Proteomics 13(8):2101-2113. comparison and statistical analysis of relative expression results in real-time PCR. Nucleic acids research 30(9):e36-e36.

25. Reddy R (2015) A comparison of methods: normalizing high-throughput RNA sequencing data. bioRxiv 026062.

26. Rueda A, Barturen G, Lebrón R, Gómez-Martín C, Alganza Á, Oliver JL et al (2015) sRNAtoolbox: an integrated collection of small RNA research tools. Nucleic Acids Res. 43:W467-73. Lugtenberg B (ed) Principles of Plant Microbe Interactions. Springer International Publishing, Switzerland, pp 371-78

28. Stam R, Jupe J, Howden AJ, Morris JA, Boevink PC, Hedley PE et al (2013) Identification and characterization CRN effectors in Phytophthora capsici shows modularity and functional diversity. PloS one 8:e59517.

29. Tani S, Judelson H (2006) Activation of zoosporogenesis-specific genes in Phytophthora infestans involves a 7-nucleotide promoter motif and cold-induced membrane rigidity. Eukaryotic cell 5(4):745-752. Small RNAs Homologous to Effector-Encoding Genes and Transposable Elements in the Oomycete Phytophthora infestans. PLoS ONE 7:e51399.

31. Yan HZ, Liou RF (2006) Selection of internal control genes for real-time quantitative RT-PCR assays in the oomycete plant pathogen Phytophthora parasitica. Fungal Genetics and Biology 43(6):430-438. 
291 32. Ye J, Coulouris G, Zaretskaya I, Cutcutache I, Rozen S, Madden TL (2012) Primer-BLAST: a tool to design target-specific primers for polymerase chain reaction. BMC bioinformatics 13(1):134.

33. Zuluaga AP, Vega-Arreguín JC, Fei Z, Ponnala L, Lee SJ, Matas AJ et al (2016) Transcriptional dynamics of Phytophthora infestans during sequential stages of hemibiotrophic infection of tomato. Molecular Plant Pathology 17:29-41. 

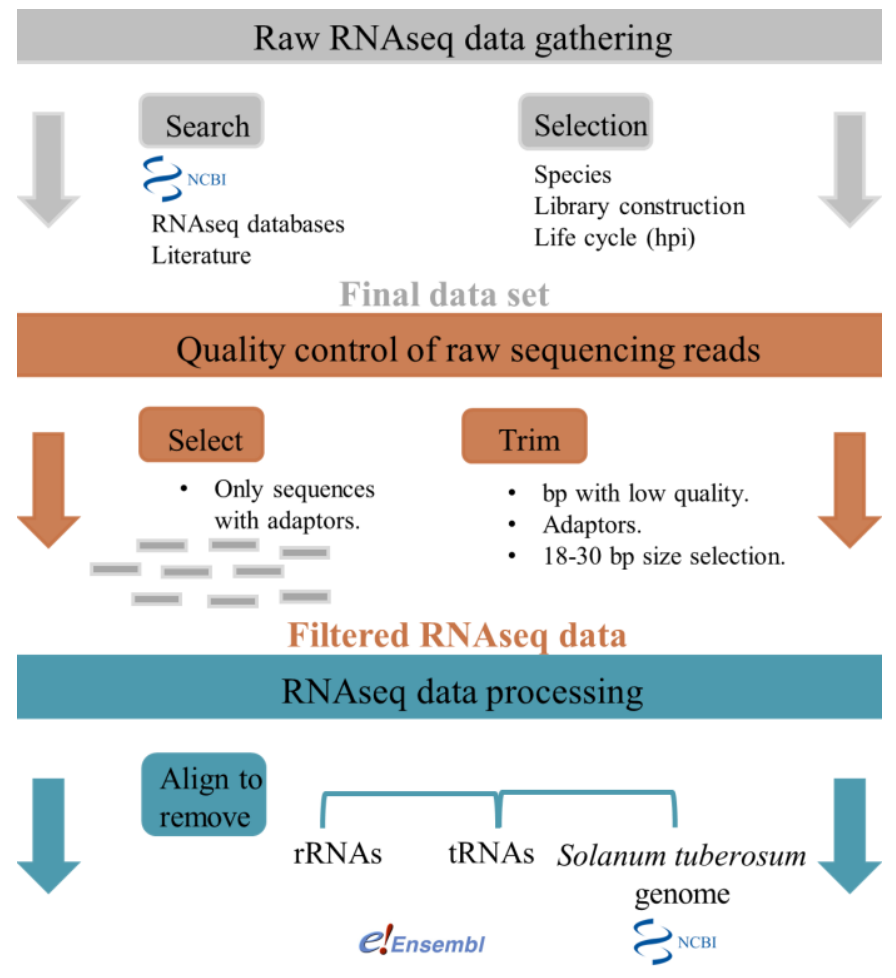

Processed RNAseq data

\section{RNAseq data analysis}

\section{Align}

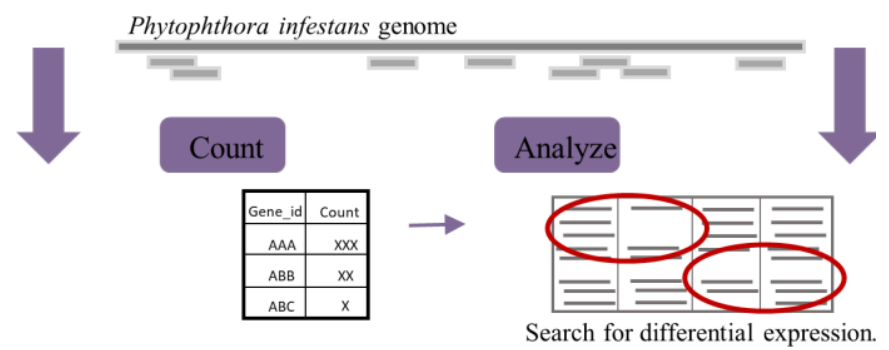

Pipeline results

322 Figure 1. Proposed methodological pipeline for the detection of small non-coding RNAs and analyses of small

323 RNA-seq data. The pipeline follows a sequential order and each colored bar represents one of the four sections of

324 this process. Figure was adapted from Maze et al. 2014. 


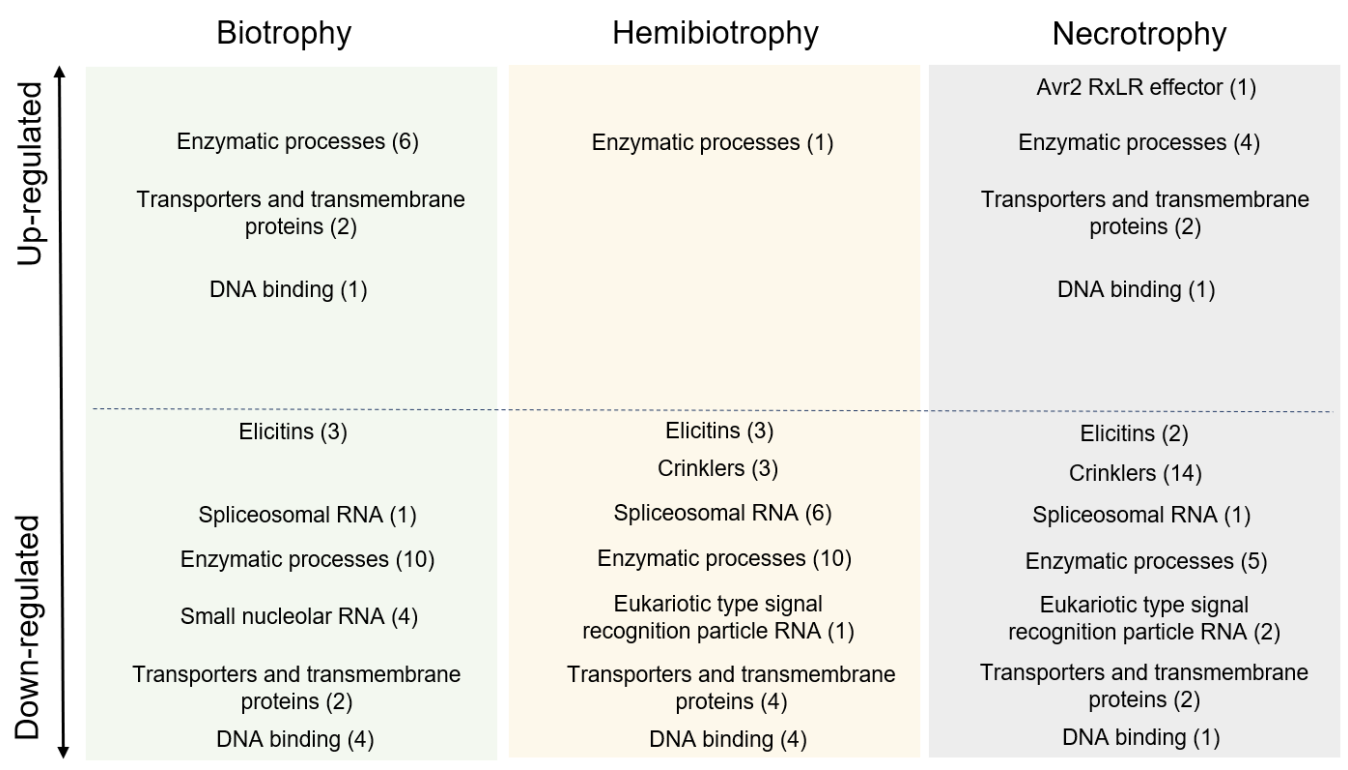

331 Figure 2. Significantly differentially expressed small non-coding RNAs in Phytophthora infestans infecting

332 Solanum tuberosum leaves were classified into nine functional categories. Up or down regulated sRNAs belonging

333 to a specific functional category at a specific time point during the cycle are shown. Each box represents one stage of

334 the infection: biotrophy (green), the transition from biotrophy to necrotrophy (yellow) and necrotrophy (grey).

335 Baseline gene expression is calculated in the in vitro mycelia, which is represented by the dotted line. Above this line

336 were upregulated, while those below the line were downregulated when compared to the in vitro mycelia. The number

337 in parentheses indicates how many sRNAs of each category were either up-regulated or down-regulated at each time

338 point. 


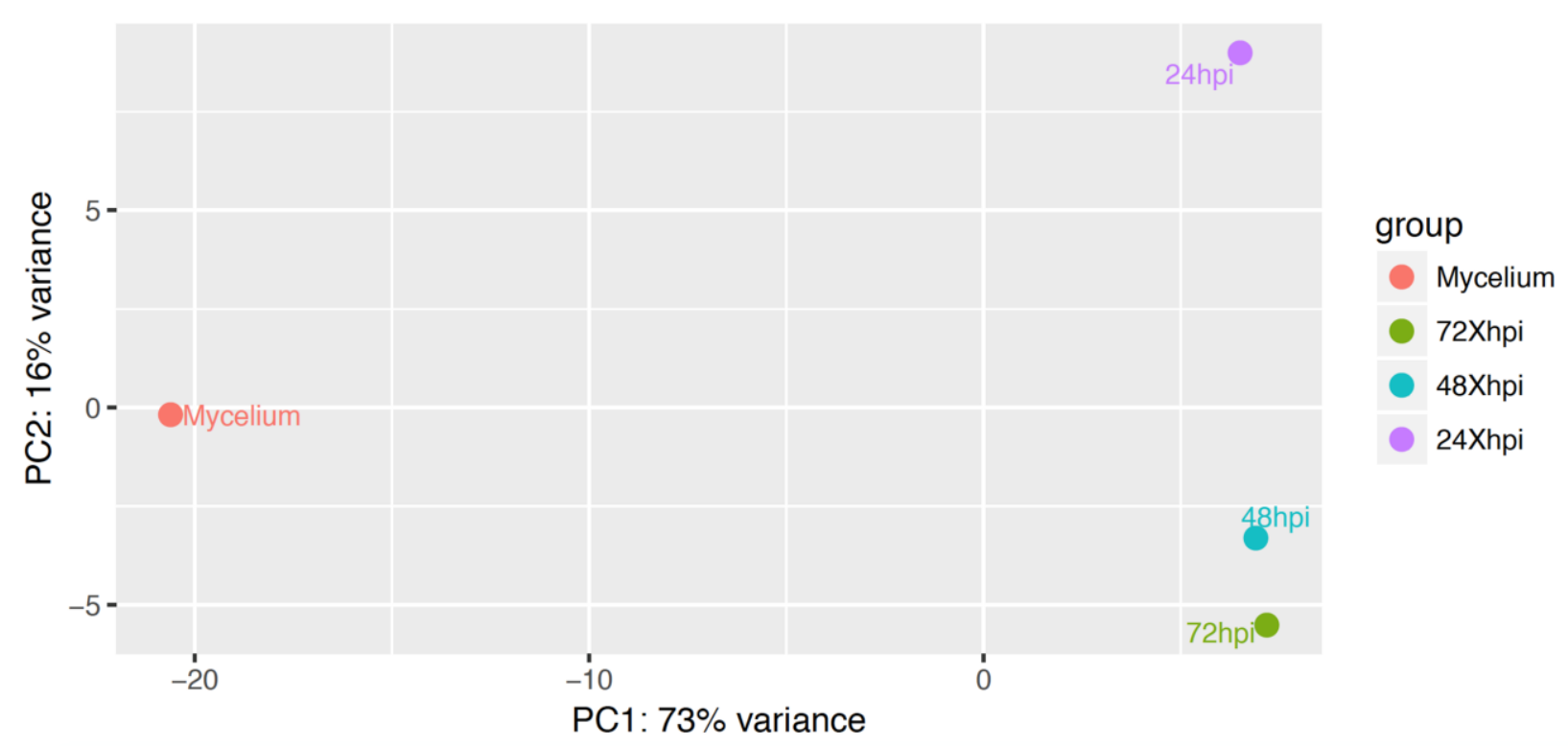

341 Figure 3. Principal components analysis (PCA) of significantly differentially expressed small non-coding RNAs

342 at each point in the infection cycle. Each dot represents sRNAs of one of the four analyzed data sets (24, 48, and 72

343 hours post inoculation and mycelia grown in culture media). The distance between them in the grid represent sample-

344 to-sample distances. In other words, how similar these data sets were between them. 


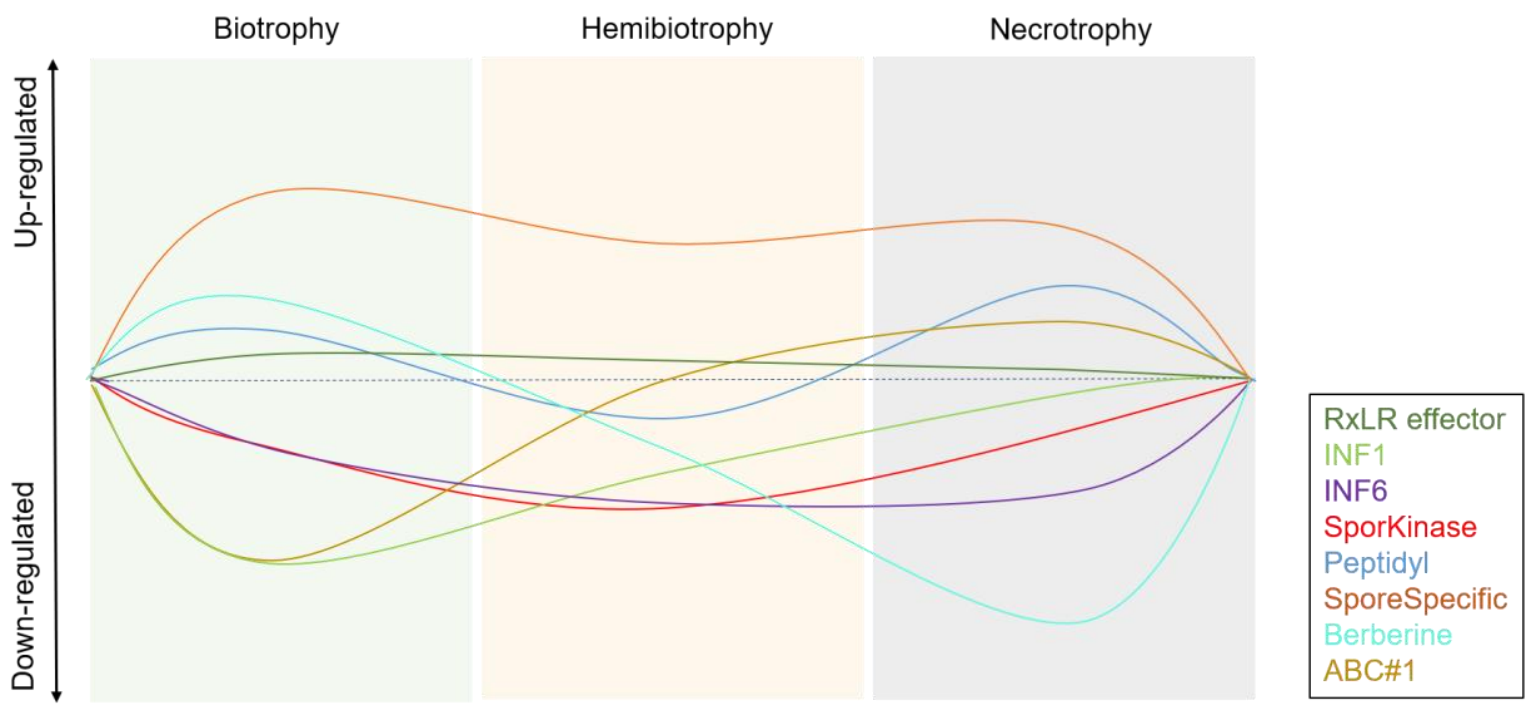

353 Figure 4. Expression levels identified by qRT-PCR of ten genes associated to relevant small non-coding RNAs

354 during the cycle. Each colored box represents one stage of the infection: biotrophy (green), the transition from

355 biotrophy to necrotrophy (yellow) and necrotrophy (grey). Furthermore, each colored line represents each of the genes

356 that were evaluated (see legend). Baseline gene expression is calculated in the in vitro mycelia, which is represented

357 by the dotted line. Genes above this line were upregulated, while those below the line were downregulated when

358 compared to the in vitro mycelia. 


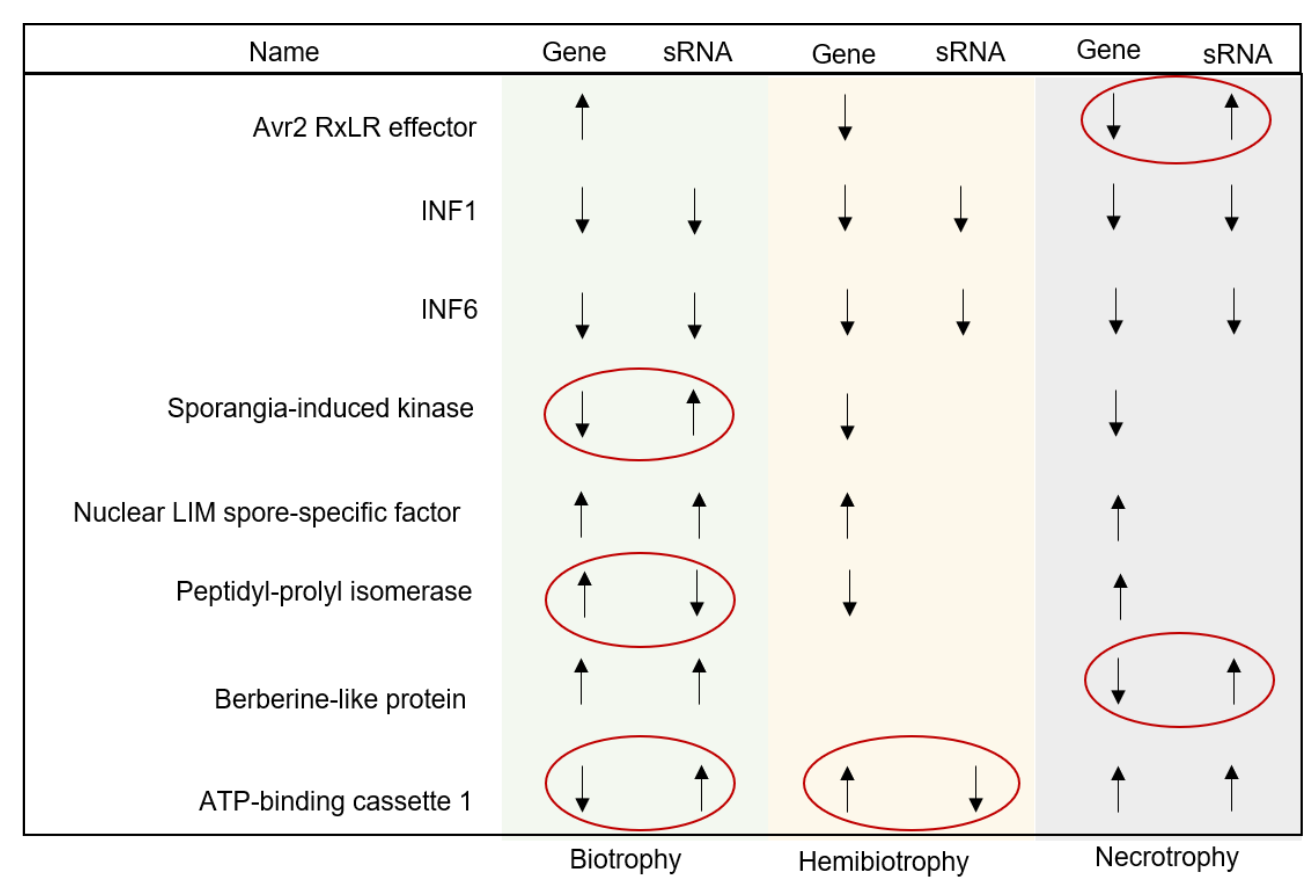

362 Figure 5. Comparison between significantly differentially expressed genes and their corresponding small non-

363 coding RNAs at different time points during the infection cycle. Eight of the ten genes that were evaluated by qRT-

364 PCR were significantly differential expressed at some point in the infection cycle. Each colored box represents each 365 of the stages in the cycle (green for biotrophy, yellow for the transition to necrotrophy and grey for necrotrophy).

366 Arrows pointing upwards represent upregulation and arrows pointing downwards represent downregulation, for both

367 the gene and its corresponding sRNA. In the cases were no arrow is present for a specific sRNA, no significant 368 differential expression was detected during that specific time point in our bioinformatic analyses. Inverse correlations 369 between the expression of a gene and its sRNA are indicated with a red circle, at relevant time points. 
370 Table 1. Genes whose expression was tested via qRT-PCR as well as the exon to exon primers designed and used for 371 these assays.

\begin{tabular}{|c|c|c|c|c|}
\hline Gene ID & Name & Category & Forward primer & Reverse primer \\
\hline PITG_04425 & $\begin{array}{c}\text { 40S Ribosomal } \\
\text { protein S3A }\end{array}$ & $\begin{array}{c}\text { Normalizer gene for } \\
\text { qRT-PCR }\end{array}$ & CTGTCCATCCAAGCGCTACT & TTGAGGAACTGCTCGTGGTC \\
\hline PITG_22870 & $\begin{array}{l}\text { Avr2 family secreted } \\
\text { RxLR effector } \\
\text { peptide protein }\end{array}$ & Avr2 RxLR effector & GCCGTGTCGATAATGGGGAA & TCGCCTTTACCGCAGCTTTA \\
\hline PITG_12551 & Elicitin (INF1) & $\begin{array}{c}\text { Elicitin and elicitin-like } \\
\text { proteins }\end{array}$ & TTCTCGTCTACGTGTGCGTC & $\begin{array}{l}\text { ACGCGGAGAACAAAGCCTA } \\
\text { A }\end{array}$ \\
\hline PITG_12556 & Elicitin-like INF6 & $\begin{array}{c}\text { Elicitin and elicitin-like } \\
\text { proteins }\end{array}$ & CTCCACTTTCGAGACGCAGT & $\begin{array}{l}\text { GAAGGAGCAGATGTAGGGG } \\
\mathrm{C}\end{array}$ \\
\hline PITG_20221 & $\begin{array}{c}\text { Sporangia induced } \\
\text { phosphatidylinositol } \\
\text { kinase }\end{array}$ & Enzymatic processes & CGCTCCGGTATCCCATACAC & GAAAAACTCGTTGGGTGGGC \\
\hline PITG_03856 & $\begin{array}{l}\text { Peptidyl-prolyl cis- } \\
\text { trans isomerase }\end{array}$ & Enzymatic processes & TCCTGCTAACGCAACACTGA & CGATCTTCTCCTCAGCGTCC \\
\hline PITG_11241 & $\begin{array}{l}\text { Nuclear LIM factor } \\
\text { interactor-interacting } \\
\text { protein spore- } \\
\text { specific form }\end{array}$ & Enzymatic processes & $\begin{array}{l}\text { AAGACGTGGAAGATGTGCG } \\
\mathrm{T}\end{array}$ & $\begin{array}{l}\text { AAGCAAGAGCTCATCGGAC } \\
\text { G }\end{array}$ \\
\hline PITG_02930 & $\begin{array}{c}\text { Berberine-like } \\
\text { protein }\end{array}$ & $\begin{array}{c}\text { Enzymatic } \\
\text { processes/DNA binding } \\
\text { or binding sites }\end{array}$ & GTTGAGACCTCGCTACCCAC & CGCATTTCAGAGTGGCAGAA \\
\hline PITG_04289 & $\begin{array}{l}\text { ATP-binding } \\
\text { cassette (ABC) } \\
\text { superfamily }\end{array}$ & $\begin{array}{c}\text { Transporters and } \\
\text { transmembrane proteins }\end{array}$ & CCGTCCTCTCATCTGTACGC & CATGTACTGCGGACACTGGT \\
\hline PITG_13576 & $\begin{array}{l}\text { ATP-binding } \\
\text { cassette (ABC) } \\
\text { superfamily }\end{array}$ & $\begin{array}{c}\text { Transporters and } \\
\text { transmembrane proteins }\end{array}$ & TCTGCTCTGATGCCCAACG & $\begin{array}{l}\text { TTGTAACCGGCCTTCATCGA } \\
\text { C }\end{array}$ \\
\hline PITG_00375 & $\begin{array}{l}\text { Haustorium-specific } \\
\text { membrane protein }\end{array}$ & $\begin{array}{c}\text { Transporters and } \\
\text { transmembrane proteins }\end{array}$ & GGGTATCTTCGGTACGCTGG & TCCGTAGTCGGGATCAAGGT \\
\hline
\end{tabular}

